\title{
La minería de carbón en Colombia y la situación económica de las mujeres rurales: la comunidad El Hatillo (Cesar, Colombia)*
}

Coal Mining in Colombia and the Financial Situation of Rural Women: The Community in El Hatillo

$$
\text { (Cesar, Colombia) }
$$

Feline Gerstenberg ${ }^{\mathrm{a}}$

Universidad Heinrich-Heine Düsseldorf, Alemania

DOI: https://doi.org/10.11144/Javeriana.ayd23-45.mccs

gerstenberg.f@gmail.com

ORCID: http://orcid.org/0000-0002-4926-0343

Recepción: 21 Abril 2019

Paula Andrea Villegas González

Aceptación: 02 Junio 2019

Universidad Católica de Colombia, Colombia

ORCID: http://orcid.org/0000-0002-9331-8979

Publicación: 30 Diciembre 2019

\section{Resumen:}

El carbón es el segundo producto más importante para el mercado de exportación de Colombia. Desde el inicio del siglo XX, los gobiernos han creado condiciones cada vez más favorables para que empresas transnacionales establezcan o amplíen sus proyectos mineros en el país. Como consecuencia, el volumen de extracción ha aumentado desde entonces. Pero mientras que el Gobierno colombiano afirmó, en el año 2012, que la explotación minera es una de las "locomotoras para el desarrollo", y datos a nivel macro evidencian lo mismo, es dudoso que solo parte de la población colombiana se beneficie de este modelo de desarrollo. Este artículo tiene como propósito responder a la siguiente pregunta: ¿hasta qué punto la minería de carbón a gran escala cambia la situación económica de las mujeres rurales del país? Con el fin de encontrar la respuesta el artículo hace énfasis en las experiencias de ellas y cuenta la realidad desde su perspectiva. Se analizaron estudios previos y fueron realizadas entrevistas cualitativas con mujeres de una comunidad que debe ser reasentada desde el año 2012: El Hatillo - Cesar, Colombia. Como resultado de la investigación, se formularon cuatro hipótesis relacionadas con los retos que la minería genera para las mujeres rurales confrontación con la destrucción de sus medios de subsistencia; alteración con su rol tradicional dentro de la comunidad y la familia; pocas posibilidades de acceder a trabajos en las minas y se presenta un alto nivel de desigualdad de género dentro del sector.

Palabras clave: minería, extractivismo, género, conflicto socioambiental.

\section{Abstract:}

Coal is the second main commodity for export in Colombia. Since beginning the 20th Century, the governments have created conditions increasingly favorable for the foreign companies to start or increase their mining projects in our country. As a consequence, the extraction volume has increased ever since. While the Colombian Government stated in 2012 that the mining exploitation is one of the "locomotives for development" and the macro level data go on this direction, it is quite worrying that only a part of the Colombian population benefits from this development model. This article aims to answer the following question: To what extent the coal mining on a large scale is changing the financial situation of the rural women in our country? To answer it, this article emphasizes the experiences by these women and tells the reality from their perspective. This work analyzed previous studies and conducted some qualitative interviews to women from a community that should be relocated since 2012: El Hatillo, in Cesar Province, Colombia. As a result from this research, four hypotheses were set out as related to the challenges the coal mining pose to those women facing the destruction of the sources for making a living, the alteration of their traditional roles both in the community and the family, scarce possibilities to access to jobs in the mines and the resulting high level of gender inequalities in this business.

Keywords: mining, extractivism, gender, social-environmental conflict.

Notas de autor

${ }^{a}$ Autora de correspondencia. Correo electrónico: gerstenberg.f@gmail.com 


\section{Introducción}

Las crisis económicas y financieras en el mundo, la producción y el estilo de vida en el norte global que dependen de recursos naturales, y la demanda de materias primas por parte de las antiguas y nuevas potencias industriales han generado el aumento de los precios de los recursos naturales, desde principios del siglo XXI.

En la literatura se habla de un "auge de la materia prima" ${ }^{[1]}$ (Burchardt y Dietz, 2012, p. 185), es decir, un desarrollo económico que se enfoca en la explotación de recursos naturales. Alrededor del $75 \%$ de la extracción mundial de materias primas se realiza en países en desarrollo y emergentes, especialmente en África Subsahariana, Asia y América Latina (Brand y Dietz, 2014).

En América Latina, se ha desarrollado un debate sobre los términos extractivismo y neo-extractivismo (Gudynas, 2013, p. 3) junto con las estrategias de desarrollo basadas en materias primas. Independiente de la orientación política de los gobiernos de la región, la cuestión es si la extracción masiva de estas materias puede generar un modelo de desarrollo sostenible que vaya acompañado de un crecimiento económico duradero y una mayor prosperidad. El Banco Mundial ha estado hablando de Industrias Extractivas (IE), refiriéndose a la exploración y extracción de petróleo y gas desde la década de 1990 (Eftimie, Heller y Strongman, 2009). Gudynas (2013) explica que la extracción de materias primas está sujeta a criterios específicos cuando se habla del (neo)extractivismo: los bienes se extraen en grandes cantidades, al menos el 50\% deben ser exportados, y no son o solo se encuentran mínimamente procesados. En Colombia, se produjeron 90,5 millones de toneladas de carbón en 2016, el número más alto históricamente (Urbina Sánchez, Cita Triana y Campo Robledo, 2016). Por lo que el Gobierno colombiano define la minería del carbón como una de las "locomotoras del desarrollo" que significa "prosperidad de todos, más empleo, menos pobreza y más seguridad" (Centro de Investigación y Educación Popular [Cinep], 2012, p. 21), citando el Plan Nacional de Desarrollo, 2011-2014. En este artículo se analiza específicamente ¿hasta qué punto cambia la minería legal de carbón en Colombia a gran escala la situación económica de las mujeres rurales?

Existe un vacío de investigación que relacione el (neo)extractivismo con una perspectiva de género. Esta brecha es cada vez más reconocida (véase, por ejemplo, Bebbington, 2015, y Jenkins, 2014). Enfocarse en las mujeres rurales ${ }^{[3]}$ es de gran importancia por tres aspectos principales: Primero (i), dada la alta tasa de pobreza de mujeres rurales en América Latina y la violación de sus Derechos Humanos (DDHH). Según la Comisión Económica para América Latina y el Caribe (Cepal) y Naciones Unidas (2016), el $23 \%$ de las mujeres rurales en la región vivieron bajo pobreza extrema en el año 2014. En Colombia, las mujeres representaban para el año 2016 el 51,6 \% de la población, el 21,2 \% de ellas vivía en zonas rurales y un $23 \%$ de los 3.070 .586 hogares rurales contaban con una jefatura femenina (Departamento Nacional de Planeación [DNP], 2018). Aproximadamente, el $45 \%$ de las mujeres rurales campesinas, indígenas y afrodescendientes de Colombia son consideradas pobres. Adicionalmente, ellas son las más afectadas por el conflicto armado que ha vivido el país.

La Plataforma de Incidencia Política de las Mujeres Rurales Colombianas nació en 2015, con el objetivo de hacer visibles sus necesidades. Ahí, ellas explican que se ven confrontadas con "profundas discriminaciones, incluido el escaso reconocimiento en términos de su participación social, política y económica, con un efecto directo en los enfoques de las políticas públicas" (YoSoyMujerRural, 2019). Entre el 18 de febrero y el 8 de marzo de 2019, se llevaron a cabo las reuniones del Comité de Naciones Unidas para la Eliminación de la Discriminación contra la Mujer (Cedaw, por sus siglas en inglés) en Ginebra, Suiza. También se revisó el estado de las mujeres rurales en Colombia. Setenta organizaciones sociales del país que trabajan por, para y con mujeres rurales y campesinas en Colombia -incluso la plataforma mencionada- crearon un primer informe alternativo que visibiliza la violación de los derechos de las mujeres rurales y campesinas, titulado Informe Sombra (Cedaw, 2019).

Este fue presentado ante el Cedaw. Marlen Alfonso, que forma parte de la plataforma de incidencia, basa su descontento con la situación actual de las mujeres rurales en Colombia en los siguientes cuatro aspectos: 
primero, la inequidad en la estructura económica pauperiza el trabajo de las mujeres rurales; segundo, el Sistema Estadístico Nacional no integra indicadores de género; tercero, el Estado colombiano restringe los mecanismos de participación de las mujeres rurales y campesinas, y en cuarto lugar, persiste una adopción de marcos legales regresivos en materia agraria y económica (Cinep, 2019).

Segundo (ii), ser mujer rural en una región minera implica una doble carga y discriminación, porque la extracción de carbón conlleva a impactos socioambientales específicos. El 76 \% de la inversión extranjera directa de Colombia se dirige a los sectores del petróleo, hidrocarburos y minería. Este hecho conlleva, según Marlen Alfonso, a una "precarización de derechos laborales, explotación sexual, servidumbre y trata de personas, afectando así la soberanía alimentaria de las mujeres rurales y campesinas” (citado por Cinep, 2019). Como se destaca en el anexo del Informe Sombra, las actividades extractivas suelen aumentar la militarización del territorio, lo que afecta el buen vivir de las comunidades y pone en riesgo a las mujeres en estas zonas. La ONU Mujeres (2019) afirma en un informe que,

las mujeres y niñas rurales influyen de manera significativa en [...] la gestión de recursos naturales, así como en el trabajo no remunerado y de cuidado doméstico. Ellas son las más afectadas cuando los recursos naturales y la agricultura se ven amenazadas.

Por el hecho de que el conflicto armado de Colombia tuviera lugar principalmente en áreas rurales, muchos habitantes de estas zonas fueron desplazados forzosamente. La restitución de tierras es un aspecto clave, incluido en el Acuerdo de Paz que fue firmado en 2016, entre las Farc-EP y el Gobierno colombiano. Pero por una política institucional que considera que las actividades extractivas no afectan el derecho a la restitución, muchas personas no pueden regresar a sus tierras, dado que estas ya se encuentran ocupadas por empresas transnacionales que extraen recursos naturales (Bautista-Revelo y Plazas-Mendieta, 2018). Ahí se encuentra otro reto para las mujeres rurales: muchas no tienen acceso a las tierras que habitaron antiguamente. Aparte de esto, la ejecución del proceso de paz es lenta e ineficiente, sobre todo con respecto a los puntos 1 y 4 . $^{[4]}$ En una entrevista que realizó la Corporación para la Vida, la Paz y el Desarrollo Sostenible con una lideresa local para conocer su percepción sobre la implementación del Acuerdo de Paz, ella cuenta que los programas como el Plan Nacional de Sustitución (PNIS) y el Programa de Desarrollo con Enfoque Territorial (PDET) no incluyen un enfoque diferencial de género y que existe una discriminación por razones de género que pone en riesgo a la mujer.

El tercer aspecto (iii) que justifica este trabajo es que la minería de carbón es un sector tradicionalmente liderado por hombres. Es del interés de esta investigación saber si las mujeres (rurales) también se benefician de la extracción de este recurso, en qué condiciones y qué alternativas existen para mejorar su situación económica. El presente trabajo es un esfuerzo para abordar la brecha investigativa mencionada y apoyar a las mujeres rurales para que sus derechos (de participación en el sector minero) se tengan en cuenta. Reconocer y empoderar a las mujeres rurales no solo es fundamental para el bienestar de ellas mismas, sus familias y las comunidades rurales, sino también generaría un impacto positivo para la productividad económica del país. De esta manera, se aumentaría la producción y el Gobierno tendría que invertir menos dinero en programas para la erradicación de la pobreza. Analizar los impactos económicos que tiene la minería de carbón para las mujeres rurales de Colombia también puede ayudar a mujeres de otras regiones de América Latina y a organizaciones sociales que trabajan con ellas. Sería útil comparar las distintas experiencias y crear mecanismos de apoyo global.

Este artículo se estructura de la siguiente manera: en primer lugar, se explica la metodología. Posteriormente, se realiza la descripción de los impactos que la minería de carbón genera para la economía de Colombia desde dos perspectivas: primero, a través de cifras macroeconómicas y, segundo, por experiencias que han vivido las propias comunidades, especialmente las mujeres. Después, se presentan los resultados que fueron obtenidos durante la estancia de investigación que se realizó en la comunidad El Hatillo y el análisis de 
estudios de otros autores. Por último, las autoras presentan una discusión sobre el tema y distintas propuestas para el abordaje y la búsqueda de soluciones a la problemática.

Es importante resaltar que este trabajo fue el producto de una estancia de investigación realizada en Colombia por la autora principal, en el marco de sus estudios de grado de Ciencias Sociales en la Universidad Heinrich-Heine, en Düsseldorf, Alemania. Durante el proceso de formación, contó con el apoyo de la Universidad Católica de Colombia, en el año 2017, como parte del semillero de investigación EcoCivil.

\section{Metodología}

La metodología utilizada en esta investigación es de corte cualitativo, ya que el objetivo fue conocer con detalle las perspectivas y experiencias de las mujeres rurales en Colombia. El análisis se divide en dos partes: primero (i), se evalúan estudios de otros autores que abordan la temática de este artículo. Segundo (ii), por el hecho de tratarse de un tema poco investigado académicamente, se realizaron entrevistas semiestandarizadas con mujeres de la comunidad El Hatillo. Para este tipo de entrevistas cualitativas se construyó una guía de preguntas, con un espectro amplio en la modalidad de preguntas abiertas o estructuradas por una teoría.

Autores y organizaciones que analizan el impacto económico de la minería legal en la vida de mujeres rurales en Colombia son, entre otros, Jazmín Romero Epiayú, de la organización Fuerzas de Mujeres Wayuu, quien publicó, en cooperación con Dana Barón Romero del Centro de Investigación y Educación Popular (Cinep), un informe sobre los impactos de la minería de carbón para mujeres en el municipio de La Guajira (2013). Otro trabajo relevante es un cuaderno de ejercicios titulado Los territorios, la minería y nosotras: las mujeres nos preguntamos. Guia de trabajo (Bermúdez Rico, Zambrano y Roa Avendaño, 2014). También se debe mencionar el informe sobre los resultados de la conferencia Encuentro Latinoamericano Mujer $y$ Minería (Bermúdez Rico, Rodríguez Maldonado y Roa Avendaño, 2011) y el estudio Mujeres, Conflictos Socioambientales y Resolución 1325 de las Naciones Unidas de Gloria Tobón Olarte (2015).

Actualmente, la mayoría de los trabajos que analizan la conexión entre la minería de carbón y mujeres rurales están escritos por organizaciones no-gubernamentales (ONG). Por esto, hay que tener en cuenta la posición subjetiva y crítica de los autores frente a la problemática. Con el fin de crear una imagen lo más objetiva y neutral posible de la situación, se realizaron entrevistas cualitativas con mujeres afectadas.

El primer paso para la selección de las mujeres entrevistadas se hizo a partir de una búsqueda de literatura. De ahí, se logró establecer que La Guajira y Cesar son las zonas mineras de carbón más importantes del país y, dado que ya existía una gran cantidad de estudios sobre la situación en La Guajira, las autoras seleccionaron al Cesar como departamento para enfocar esta investigación. La comunidad El Hatillo fue escogida para el análisis, debido a su cercanía a una de las minas de carbón a cielo abierto más grandes de Colombia. Además, porque ya había estudios que demostraban los efectos negativos que la mina genera en la salud de esta población.

El segundo paso en la selección fue ponerse en contacto con la comunidad rural. Debido a la situación de seguridad en Colombia, especialmente en las áreas rurales, era importante realizar las entrevistas en compañía de una persona que conociera las circunstancias actuales de seguridad en dicha comunidad. El así llamado Schneeballprinzip ${ }^{[5]}$ (Kruse, 2015, p. 251) demostró ser exitoso para este objetivo. El contacto clave fue una exempleada de la ONG colombiana Pensamiento y Acción Social (PAS). Ella estableció el contacto con dos personas de la ONG suiza CoMundo, que trabajaban en El Hatillo durante la época de la investigación. Con su apoyo, se organizó una estadía en la comunidad durante cuatro días. En el mes de mayo de 2017, se realizaron entrevistas a cinco mujeres de la comunidad, de carácter semiestructurado y centradas en los problemas. Con el fin de conocer diversas experiencias y perspectivas, se entrevistó a un panorama amplio de mujeres rurales. La selección se basó en los siguientes criterios: primero (i), era indispensable que las mujeres pudieran comparar la situación del pasado, es decir, antes de la llegada de las compañías mineras con la 
situación actual. Segundo (ii), fue importante que las mujeres tuvieran diferentes edades, lo que permitiría un análisis diverso de la realidad. Se eligieron mujeres que tenían entre 34 y 64 años, a la hora de realizar las entrevistas. Un tercer aspecto (iii) era la inclusión de mujeres que tuvieran experiencia laboral en la minería, o que su pareja la tuviera. Esto era fundamental para conocer las realidades laborales dentro del sector minero. Por último, se quería incluir la voz de por lo menos una mujer lideresa de la comunidad. A continuación, se presentará una breve caracterización sociodemográfica de las mujeres entrevistadas, con el objetivo de analizar luego sus testimonios en contexto y a la luz de sus circunstancias particulares.

- Mujer 1: 36 años, nació en El Hatillo, pero ha vivido fuera de la comunidad por 10 años y tiene 8 niños. Hace unos años, ella criaba animales (cerdos y gallinas), pero no pudo seguir porque una peste los mató. Dice que esta es la causa principal de su mala situación económica, junto con la pérdida de tierras. Tiene que salir del pueblo para buscar trabajo.

- Mujer 2: 45 años, creció en Codazzi (Cesar) y lleva aproximadamente 20 años en El Hatillo. Tiene 5 niños y su pareja trabaja en la minería como mecánico con un contrato de 6 meses. Esta circunstancia dificulta conocer su situación económica futura. Ella no trabaja, antes era "ama de casa". No quiere trabajar en la mina por los impactos negativos que genera para la salud. Ella cuenta que un primo trabajó durante 3 años para una empresa minera como camionero y ahora tiene problemas de columna por un accidente durante el trabajo, por lo que la empresa no lo puede recibir más. Una de sus hijas hizo un curso de administración que ofreció la empresa Prodeco, duró un año, pero según la madre no ha conseguido trabajo.

- Mujer 3: 64 años, nació en El Hatillo, ha vivido un tiempo afuera y volvió hace 13 años a la comunidad. Ella tiene 4 niños y es madre soltera. Antes de la llegada de la minería, cortaba leña y hacía galletas. Hoy en día no es posible porque no tiene un horno. Por causa de la extracción minería se destruyó su casa ("me tumbaron la casa y se me fue el suelo, las paredes se le cayeron, quedé en el aire, no tenía casa”). Después de 2 años construyó la casa nuevamente.

- Mujer 4: 39 años, nació en San Juan de la Guajira y creció en Valledupar. Allí estudió y vino hace 19 años a El Hatillo. Su pareja trabaja en la minería y ella mantiene una peluquería en la comunidad. Es una de las lideresas de El Hatillo.

- Mujer 5: 34 años, creció en El Hatillo y tiene 4 niños. Ella no tiene trabajo. Antes de la llegada de la minería, cultivaba.

Se usó una muestra limitada de mujeres, con el objetivo de conocer ciertas perspectivas con detalle, en vez de obtener una gran cantidad de voces. Sin embargo, se recomienda considerar una diversidad mayor en una investigación futura. Por ejemplo, mujeres que forman parte de la comunidad LGBT o mujeres con discapacidad que viven en áreas rurales. Las entrevistas se llevaron a cabo en la comunidad El Hatillo, en las casas de las mujeres o afuera de estas. Solo a la Mujer 4 se le hizo la entrevista en su peluquería, mientras trabajaba. La duración promedio de las entrevistas era de 45 minutos a una hora. Cada entrevista se realizó durante una única sesión.

La guía de entrevista utilizada se creó con el modelo "SPSS” que desarrolló Cornelia Helfferich (2011). En primer lugar, se construyó una lista con diferentes preguntas, antes de verificarlas, clasificarlas y finalmente ordenarlas en las cuatro categorías "estímulo", "contenido", "pregunta de ayuda" y "respuestas concretas" (Helfferich, 2011). Preguntas claves que guiaron las entrevistas fueron: ¿Usted siente que la explotación minera de carbón ha tenido un impacto positivo en el país y su gente? ¿En qué trabajó usted antes de la llegada de la minería y en qué trabaja hoy? ¿Usted ha experimentado que la llegada de las empresas mineras ha generado nuevas oportunidades de ingreso/trabajo para las mujeres de su comunidad?

Las entrevistas fueron grabadas con un dispositivo electrónico. Todas las mujeres habían dado previamente su consentimiento. Para su seguridad, no se van a mencionar sus nombres. Para el procedimiento de las entrevistas fue usada la técnica Análisis Cualitativo de Contenido, la cual es catalogada como exitosa, 
en cuanto extrae las expresiones y temas más importantes del conjunto de datos. Con esto se pretende crear una intersubjetividad (Blatter, Janning y Wagemann, 2009). Sin embargo, por razones pragmáticas de investigación, no parecía útil adherirse a todos los pasos del análisis de datos.

\section{El sector minero de Colombia}

Desde el año 2001, la importancia del sector extractivo en Colombia ha crecido. Esto se ilustra considerando los siguientes indicadores macroeconómicos: el porcentaje de bienes primarios en el producto interno bruto (PIB) aumentó de 5,7 \%, en el año 2000, a 7,7 \% en el 2012 (Olivares Rivera y Manrique Galvis, 2018). Con una participación del 14,9\% de las exportaciones totales, el carbón fue el segundo producto de exportación más importante del país después del petróleo, en el año 2016 (Cepalstat, 2017). La importancia del carbón para la economía de Colombia también se refleja en las cifras de producción: en 1985 el país produjo 8,98 millones de toneladas, en 2016 alcanzó su máximo histórico de 91,08 millones de toneladas (Unidad de Planeación Minero Energética [UPME], 2018). Parece evidente entonces por qué el Ministerio de Minas y Energía (Minminas) y la Agencia Nacional de Minería (ANM) (2017) describen la minería del carbón como una "fuente importante de desarrollo y economía" (p. 1).

La importancia del carbón para la economía del país está relacionada con "coyunturas de precios internacionales en el mercado energético y con los incentivos que el Estado otorga para la inversión en el sector" (Observatorio de Conflictos Ambientales [OCA], 2019). Aparte de esto, tiene que ver con las políticas y el marco legal minero, el cual ha cambiado significativamente en los últimos años. Las normas generales son la Ley 2 de 1959, sobre la economía forestal de la Nación y conservación de recursos naturales renovables, y la Constitución Política de 1991, con sentencia c-595 de 2010 que regula la relación entre la sociedad colombiana y la naturaleza, buscando proteger el medio ambiente. El artículo número 8 contempla una obligación no solo del Estado, sino también de las personas a ejercer dicha protección. La Ley 99 de 1993 crea el Ministerio de Medio Ambiente y reorganiza las entidades encargadas de su gestión. El código de minas, la Ley 658 de 2001, trata de la propiedad estatal, el derecho de explorar y explotar, entre otras. A nivel nacional, hay poca claridad sobre las áreas excluidas para el desarrollo de actividades mineras. Según el artículo 34 de la Ley 685 (2001) existen estas áreas y podrán declararse, pero en la realidad se hacen evidentes inconsistencias cuando una empresa minera proyecta actividades extractivas, en zonas de reserva ambiental. En este caso sustrae dicha zona y deja de ser excluida (Bohórquez Rozo, 2014).

Para entender bien la estructura y la organización del sector minero de Colombia, hay que mencionar las instituciones más importantes y el rol del Estado. El Plan Nacional de Desarrollo Minero - Visión 2019, que se publicó en 2006, explica que el Estado colombiano no solamente desempeñó funciones administrativas en el sector, sino que también actuó como empresario minero. Se puede decir que su función, desde entonces, se concentra en la facilitación de la extracción y producción de carbón (UPME, 2006). Estas funciones también las asignó la Ley minera del año 2001. El Plan Nacional de Desarrollo 2010-2014 (DNP, 2011) marcó esta posición del Estado como uno de los principios del modelo de desarrollo colombiano: "El mercado hasta donde sea posible y el Estado hasta donde sea necesario" (p. 16). Según la Ley minera, el Estado tiene la obligación de: "Procurar que quienes operan el negocio minero lo hagan con criterios de desarrollo sostenible, de tal manera que generando riqueza para los empresarios mineros puedan contribuir al bienestar de las comunidades involucradas y al progreso de la economía nacional" (UPME, 2006, p. 13). Sin embargo, no hay suficientes evidencias para demostrar si el Estado realmente cumple con esta obligación. Los indicadores que muestran que el sector minero de Colombia ha subido en importancia a un nivel macro no visibilizan los impactos para el nivel micro, es decir, para las comunidades. Hay muchos autores que le critican al Estado colombiano que no distribuye de manera justa las ganancias que recibe por la extracción de carbón en toda la sociedad, sino que solamente se beneficia una parte marginal. Otro aspecto para considerar es la toma 
de decisiones sobre proyectos mineros, como lo afirma Bohórquez Rozo (2014) las comunidades negras e indígenas tienen pocas posibilidades para participar en los procesos de toma de decisiones sobre la explotación de una zona: "[...] su opción es explotar o permitir que otros lo hagan con los posibles recursos existentes, no hay posibilidad de negarse a pesar de las potenciales consecuencias ambientales [...]” (p. 6).

Minminas es la máxima autoridad del sector minero colombiano. Su responsabilidad es administrar los recursos naturales no renovables del país (energéticos y mineros) y asegurar que se usen de la mejor manera. Aparte de esto, se encuentra encargado de la regulación y el uso de estos recursos, y debe garantizar su abastecimiento protegiendo el medio ambiente. Otra institución importante del sector minero es la Unidad de Planeación Minero Energética (UPME), adscrita al Ministerio de Minas y Energía, y regida por la Ley 143 de 1994 y el Decreto número 1258 de junio 17 de 2013. Su rol es principalmente la planificación, el desarrollo y el aprovechamiento de los recursos minero-energéticos de una forma integral, indicativa, permanente y coordinada con los agentes del sector minero.

El Servicio Geológico Colombiano (SGC) (anteriormente, Ingeominas) debe realizar investigaciones sobre los recursos del subsuelo, adelantar y monitorear las amenazas del origen geológico y administrar la información sobre el subsuelo. Además, debe garantizar la gestión segura de los materiales nucleares y radiactivos de Colombia. A inicios de 2011, el SGC recibió críticas por su función en el sector extractivo. Se le diagnosticó una debilidad institucional. El Gobierno ha venido fortaleciendo la institucionalidad, cambiando el marco legislativo, a través del Decreto 4134 (noviembre 4 de 2011) se creó la Agencia Nacional de Minerales (ANM), máximo órgano para el manejo de la política minera.

Como ya se ha mencionado antes, las regiones carboníferas más importantes de Colombia son La Guajira y Cesar. Esto se debe a tres razones, según Hawkins (2014): primero, porque tienen un alto nivel de producción. Segundo, porque el carbón se extrae a cielo abierto, lo que implica el uso de tecnologías profesionales y, en tercer lugar, Cesar y La Guajira están geográficamente cerca de los puertos más relevantes del país (Ciénaga y Santa Marta). Como resultado, el transporte de carbón es más barato, en comparación a si se trajera desde el interior. Las empresas de carbón más importantes actualmente son Cerrejón y Drummond. Cerrejón es una antigua fusión entre el Estado colombiano (Carbocol) y la petrolera estadounidense Exxon (Intercor), ha producido 16.056 millones de toneladas de carbón en el Cesar, en 2018, casi el medio de la producción total en este departamento (UPME, 2018). Hoy en día, la compañía es propiedad del grupo de materias primas australiano-británico BHP-Billiton, de la compañía minera británica Anglo-América y del grupo empresarial suizo-británico Glencore-Xstrata. Además de Cerrejón y Drummond, las compañías Prodeco y Colombian Natural Resources (CNR) (5304 miles y 1714 miles de toneladas producidas en el Cesar, en 2018) están extrayendo mucho carbón cada año en Colombia. CNR es una empresa de carbón de Colombia, adquirida en agosto de 2015 por la empresa estadounidense Murray Energy Corp del banco de inversión Goldman Sachs (Olivares Rivera y Manrique Galvis, 2018). Prodeco es una subsidiaria de Glencore que se fusionó con Xstrata, en 2013, y tiene 337 millones de toneladas de reservas de carbón (Hawkins, 2014).

Debido al enfoque de este estudio, a continuación, se presentan algunas generalidades del departamento del Cesar. Este incluye 25 municipios (Sociedad Geográfica de Colombia [Sogeocol], 2017). A principios de los años 80, los habitantes del municipio de La Jagua de Ibirico descubrieron depósitos de carbón de piedra, minaron el carbón en pequeñas cantidades y lo usaron como combustible para sus estufas. La empresa Carbones del Caribe fue una de las primeras en extraer el carbón de manera más profesional. Drummond finalmente abrió la primera mina de carbón en Cesar, nombrada "Loma-Pribbenow", y en 1994 comenzó la producción en cantidades relativamente pequeñas (González Serrano, 2013). En los años siguientes, el distrito administrativo se convirtió cada vez más en una región próspera de carbón. Se instalaron nuevas minas y el volumen de extracción subió constantemente. Actualmente, se considera al Cesar como la región carbonífera más importante del país. En 2014, el $48 \%$ del carbón del país se estaba extrayendo en este departamento, lo que significaba alrededor de 35 millones de toneladas anuales. Según el Cinep (2014), la empresa Drummond controlaba el 60 \% de la producción. 
La figura 1 muestra la extensión del área minera del carbón en Cesar, del año 2011. A la izquierda, se pueden ver los títulos de minería válidos de este año, las áreas marcadas en rojo en la imagen de la derecha representan los títulos solicitados.

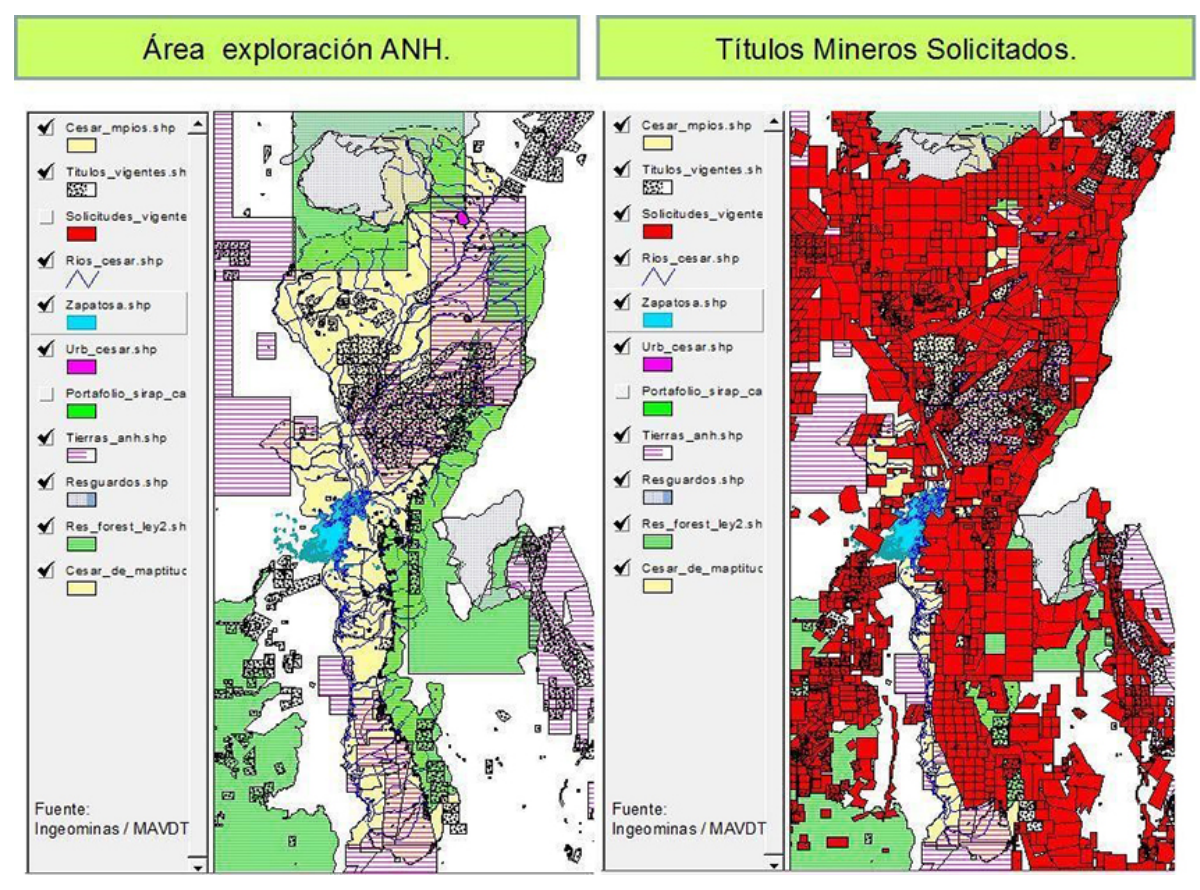

FIGURA 1.

Área de exploración y títulos mineros solicitados en el departamento del Cesar, en 2011 Fuente: Osorio (2011)

La extracción de carbón a gran escala aparece como una manera exitosa para promover la economía en Colombia. Pero como ya se ha mencionado, los indicadores económicos a nivel macro no necesariamente reflejan los impactos que genera para las comunidades. Con la esperanza de empleo, muchos colombianos y personas de fuera emigran a las áreas mineras rurales del país: el municipio La Jagua de Ibirico, por ejemplo, aumentó su población, desde 1980 hasta principios de los 90, de 8000 a 20.000 personas. El rápido crecimiento de la población ha sobrecargado los servicios públicos, aumentado la prostitución y el crimen. Hawkins (2014) muestra que las compañías de carbón internacionales ofrecen pocos empleos para el pueblo colombiano y que las condiciones de trabajo son a menudo precarias: en 2013, la proporción de mineros en la fuerza laboral total en Colombia era de solo 1,1 \%. Cerca de 30.000 personas trabajaban en las 5 principales minas de carbón. En agosto de 2014, 14.668 personas fueron contratadas en la mina El Cerrejón. Solo el 43 $\%$ de ellos tenía un contrato de trabajo, el resto era contratado por organizaciones o individuos terceros que reclutan personal para las empresas mineras. El trabajo conlleva a una serie de riesgos para la salud. Hawkins (2014) distingue entre accidentes de trabajo y enfermedades que pueden ocurrir como resultado de trabajar en la minería del carbón. Estos incluyen, por ejemplo, osteoartritis, sordera o mala postura.

\section{Descripción de la comunidad El Hatillo}

El Hatillo es una comunidad rural ubicada en el centro del Cesar, corregimiento La Loma, municipio de El Paso (figura 2). Se encuentra asentada sobre terrenos baldíos de la nación, desde hace más de 150 años. Las comunidades campesinas asentadas tienen la condición legal de ocupantes de baldíos, las cuales son reconocidas por el Estado colombiano a través de la Ley 160 de 1994 o Ley de Reforma Agraria (Cinep, 
2014). En 2011, vivían unas 190 familias en El Hatillo, que ocupaban aproximadamente 200 hectáreas de tierra (Holguín, 2011).

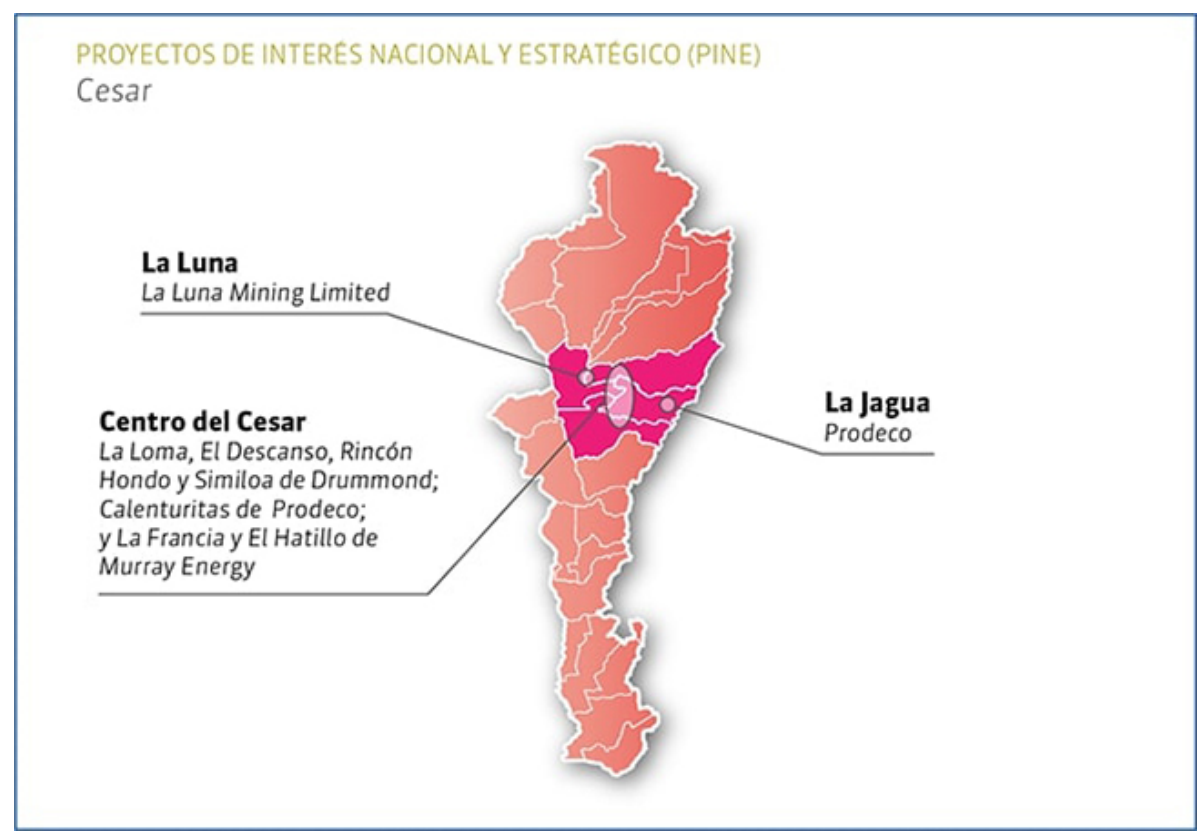

FIGURA 2.

Mapa con los proyectos de interés nacional y estratégico en el Cesar. Minas La Francia y El Hatillo Fuente: Ministerio de Minas y Energía (Minenergía) (2019)

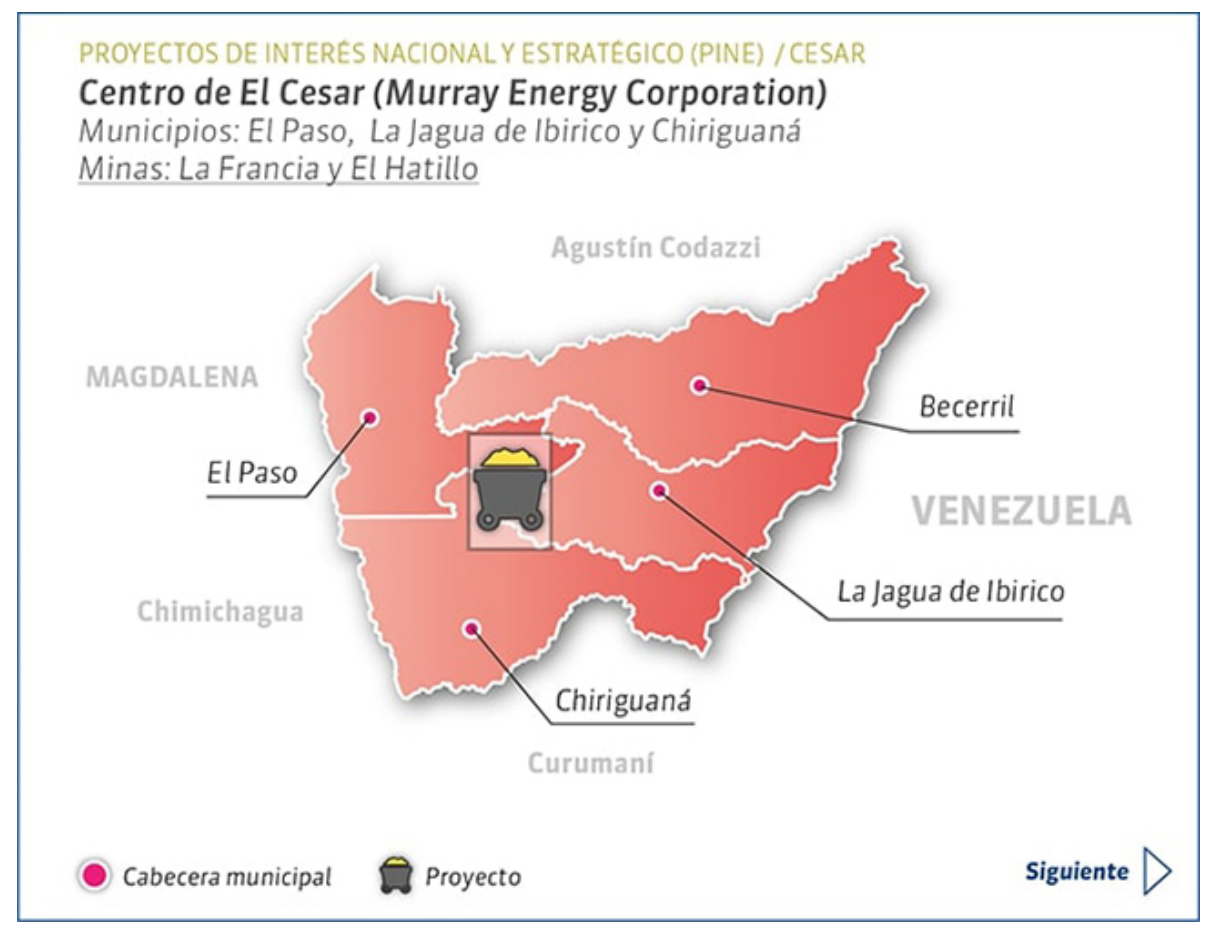

FIGURA 2.

apa con los proyectos de interés nacional y estratégico en el Cesar. Minas La Francia y El Hatillo (cont.) Fuente: Ministerio de Minas y Energía (Minenergía) (2019)

La comunidad está situada al lado de la mina que lleva el mismo nombre. Se encuentra en la región centro-sur del departamento del Cesar. Según los datos del Grupo de Trabajo Suiza-Colombia (ASK o ask en su abreviación alemana), en 2011, cuatro corporaciones mineras internacionales operaban cerca de la 
comunidad, extrayendo carbón en grandes cantidades. La compañía CNR operaba las minas El Hatillo y La Francia. Glencore / Prodeco poseían el proyecto "Calenturitas" y la compañía Drummond era el propietario de la minería a cielo abierto Descanso Medio (Arbeitsgruppe Schweiz Kolumbien, 2011).

El tamaño de las minas de El Hatillo y La Francia es de 9672 ha en conjunto. El proyecto de explotación de carbón térmico durará hasta el año 2036 y, según Minminas, cuenta con reservas de 140 millones de potencial. En 2013, trabajaban 302 trabajadores directos y 654 trabajadores contratistas (Olivares Rivera y Manrique Galvis, 2018). Las figuras 3 y 4 muestran que la comunidad El Hatillo vive a pocos metros de la mina de carbón del mismo nombre.

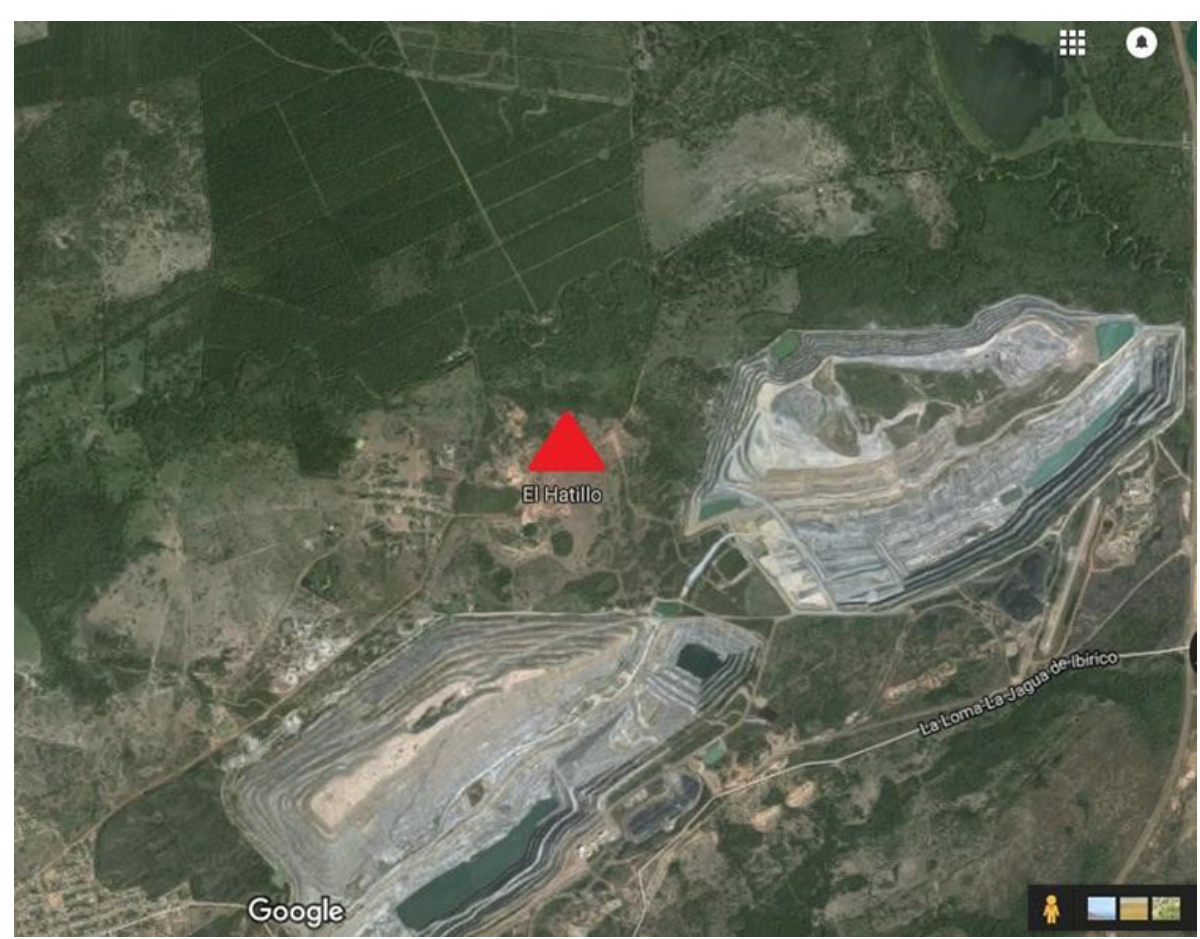

FIGURA 3.

Comunidad El Hatillo y la minería del mismo nombre Fuente: Google Earth (2019) 


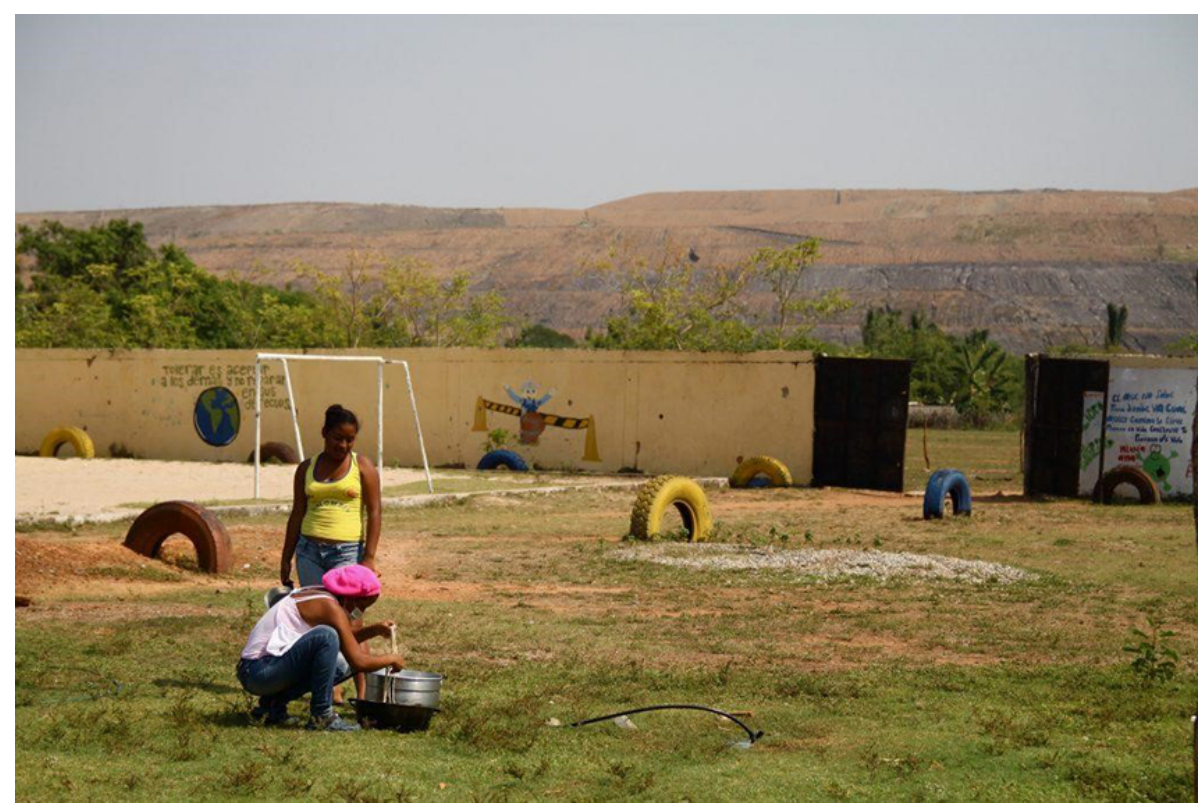

FIGURA 4.

La comunidad y la minería de El Hatillo

Fuente: Fuentes (2014)

La minería del carbón ha transformado la vida cotidiana de los así llamados hatillanos de muchas maneras, en los últimos 20 años. Desde la fundación de la comunidad hasta la llegada de las compañías de carbón la gente era autosuficiente. Es decir, pudieron vivir de lo que ellos mismos cultivaron y produjeron. Cada familia en El Hatillo tenía aproximadamente 25 hectáreas de tierra que utilizaban para fines agrícolas, antes de la llegada de la minería del carbón. El Instituto Colombiano de Desarrollo Rural (Incoder) estableció que cada familia en el departamento del Cesar debe tener al menos 35 ha para la actividad agrícola, pero las familias de El Hatillo ahora solo poseen 1,5 ha (cada una). La razón de esto es que Minminas vendió tierras de los Municipios de El Paso, La Jagua de Ibirico y Becerril, que anteriormente eran agrícolas, a empresas de carbón (Holguín, 2011). Ante la contaminación ambiental y las consecuencias para la salud de la población local, más de la mitad de los miembros de la comunidad padecen una enfermedad respiratoria, cutánea o enfermedades oculares, relacionadas con la minería. En 2010, el entonces llamado Ministerio de Ambiente, Vivienda y Desarrollo Territorial solicitó el "reasentamiento involuntario" de tres poblaciones del corredor carbonífero: Plan Bonito, El Hatillo en jurisdicción del municipio El Paso, y la población de Boquerón en jurisdicción del municipio de La Jagua. Este reasentamiento se basaba en el hecho de que los niveles de material particulado de carbón en la atmósfera habían sido más altos que los límites máximos permisibles y por eso ponían en riesgo la salud de las personas (Prodeco, 2019). Según información de Drummond (2019), en febrero de 2014 se empezó a ejecutar el Plan de Acción para el Reasentamiento (PAR) para la población de Plan Bonito; 172 de las 174 familias han sido reasentadas individualmente. En los casos de Boquerón y El Hatillo, el reasentamiento se ha convertido en un proceso muy lento y complejo.

Según un informe de Drummond (2019), en Boquerón hay dificultades por intereses divergentes dentro de la comunidad, y las personas todavía esperan su reasentamiento. Después de diálogos intensos entre distintos actores como las ONG, la población, las compañías carboníferas y el Estado, ellos firmaron el PAR para El Hatillo el 29 de noviembre 2018 (Suhner, 2019). Stephan Suhner, que forma parte de la organización ASK, considera que este paso es muy importante, pero también explica que todavía falta mucho trabajo antes de que la comunidad sea reasentada finalmente. El calendario para la implementación del PAR tiene un plazo máximo de cinco años. La ONG Comundo publicó el 14 de noviembre de 2019 fotos en su página de 
Facebook que demuestran un bloqueo de la vía del tren de carga que transporta el carbón. Las personas exigen el cumplimiento del PAR ${ }^{[6]}$.

\section{Resultados}

Este apartado tiene el objetivo de presentar los resultados obtenidos a través del análisis de investigaciones anteriores y de las entrevistas con las mujeres de El Hatillo. Dichas entrevistas fueron analizadas bajo las circunstancias particulares de cada mujer. Se presentan cuatro hipótesis con respecto a la pregunta principal de la investigación. El gran valor de este artículo es que permite conocer el fenómeno del neo-extractivismo, enfocándose en la extracción de carbón desde el género. Se visibiliza cómo las mujeres son afectadas de forma distinta a los hombres y qué retos especiales enfrentan ellas.

\section{Devaluación del trabajo}

La primera hipótesis es que la llegada de la minería de carbón conlleva una devaluación de las actividades (económicas) tradicionales de las mujeres rurales en Colombia, un cambio referido al papel de ellas dentro de su familia y la comunidad, así como una afectación negativa de su situación económica. Bermúdez Rico et al. (2011) afirman, en su estudio con referencia a la región de La Guajira, que un empleo remunerado es normalmente de baja prioridad en las comunidades rurales de Colombia, ya que los habitantes se alimentan en gran parte de lo que cultivan o crían ellos mismos. Este fenómeno se llama economía de subsistencia. Tradicionalmente, es la responsabilidad de la mujer rural asegurar la alimentación y cuidar la salud de su familia. Estas obras se denominan trabajos de cuidado. García (2012) los define como un "trabajo realizado principalmente por mujeres, cuyo objetivo es la satisfacción de las necesidades [...], que se caracteriza por la realización de múltiples tareas al mismo tiempo y por la polivalencia de los conocimientos necesarios" (p. 11).

En las comunidades rurales existe una así llamada división sexual de trabajo. Esto quiere decir que los hombres hacen trabajos remunerados, visibles y asociados al ámbito público, mientras que las mujeres los del cuidado, gratuito, invisible y asociado al ámbito privado. Hay una jerarquía donde se considera que el trabajo remunerado es más importante que el del cuidado. Este hecho conlleva a que las mujeres se encuentran en una situación menos apreciada (García, 2012). Para apoyarse entre sí en momentos de necesidad, los hatillanos realizaban trueques antes de la llegada de la minería. La Mujer 4 (lideresa de la comunidad 3 de mayo de 2017) cuenta: "Mucho antes atrás yo tenía un vecino y yo compraba arroz y él pescaba. Entonces yo iba y le ofrecía el arroz para que me diera pescado".

Según las mujeres entrevistadas, por las actividades extractivistas se ha limitado este comportamiento de solidaridad. Los trueques se han convertido en una rareza, debido a que algunos alimentos ya no están disponibles. Esta observación también la han hecho Romero Epiayú y Barón Romero (2013) en La Guajira.

Las mujeres de El Hatillo afirman que económicamente les iba bien antes de la llegada de las compañías de carbón, refiriéndose sobre todo a la seguridad alimentaria. La Mujer 3 (4 de mayo de 2017) cuenta: "[Para] el almuerzo, se iba una persona contando no tengo nada veci, voy para el río. Y traía de allá un poco de pescado. Personas que vivían de casería salían para el monte y cuando regresaban, regresaban con 2, 3 conejos en la mano, ya para comer con su familia”.

Se puede concluir que para ellas una buena situación económica significa poder alimentar a su familia y a ellas mismas. No relacionan lo económico con el dinero, sino con la garantía de sus medios de subsistencia. Sin embargo, parece que esta relación referida al dinero está cambiando. Antes, las mujeres de El Hatillo trabajaban en la agricultura, se dedicaban a la ganadería, a la caza o la pesca (Bermúdez Rico et al., 2011). Como consecuencia de la llegada de la minería y los impactos que la extracción de recursos naturales tiene para el medio ambiente, los trabajos de cuidados ya no se pueden realizar igual que antes. Por esto, los cambios 
en el uso de la tierra y los regímenes de acceso, así como la fuerte contaminación ambiental que conlleva la minería del carbón, tienen un impacto negativo para las mujeres en particular: para ellas, su papel tradicional de cuidadora se transforma en un desafío, debido a que la minería del carbón destruye importantes fuentes de alimentación. Las mujeres hatillanas afirman que la cosecha ha disminuido desde la llegada de las compañías de carbón, porque contaminan el suelo con el herbicida glifosato. Aparte de esto, las empresas mineras desvían ríos, con el fin de usar el agua para la extracción. Esto no solamente significa que la biodiversidad en los ríos disminuye y que hay menos posibilidades de pescar, sino también que las mujeres tienen menos espacios de encuentro e intercambio. Como lo consta la Mujer 4, las mujeres de su comunidad se encontraban antes al lado del río para limpiar la ropa, cantar y hablar. Según ella, el río era un espacio de encuentro y de intercambio para las mujeres:

Ellas se juntaban [...] y mientras que el marido pescaba, ellas lavaban y ellas cantaban. Era una tradición y los niños jugaban en la vía del río. Ya aquí no hay eso, si vamos al río ésta muy feo [...]. Tenían una canción que se llama el son de manduco, la ropa era el sonido de la canción y eso se perdió. (3 de mayo de 2017)

Aquí se puede observar que la minería también tiene un efecto negativo para el tejido social de la comunidad y su cultura. Se observa que es especialmente difícil para mujeres mayores encontrar un trabajo remunerado desde la llegada de la minería. La Mujer 3, que tiene 64 años, cuenta que anteriormente hacía galletas y cortaba leña. Su casa fue destruida por las explosiones de las actividades mineras y tenía que reconstruirla de nuevo. En la entrevista cuenta que no tiene el dinero para comprar un horno, algo que necesita para seguir con su labor antiguo. Actualmente trabaja con "plata de interés", como lo llama ella, es decir con dinero prestado. Según ella, es mucho más fácil para los jóvenes conseguir un trabajo en la minería: "Pero ya yo qué voy a trabajar si tengo 64 años, tengo que trabajar desde mi cuenta, lo poquito que recibo es asi”" (4 de mayo de 2017).

Otro aspecto que dificulta el aseguramiento de la subsistencia es la subida de precios. La llegada de muchos trabajadores del exterior lleva a un incremento de los precios de los alimentos en las regiones mineras. Según una de las personas entrevistadas, la mitad de un kilo de arroz solía costar alrededor de mil pesos y ahora cuesta el doble. Por no tener las posibilidades de sustentarse independientemente, las mujeres se ven forzadas a comprar estos productos. De esta manera, se ven afectadas por múltiples factores que dificultan el cumplimiento de su función como cuidadoras. Como consecuencia, se observa el surgimiento de la "pluriactividad" en el medio rural, donde las mujeres incursionan en actividades no agrícolas para generar ingresos (Farah y Pérez, 2004).

\section{Búsqueda de un trabajo remunerado}

La segunda hipótesis es que la llegada de la minería conlleva a la búsqueda de un trabajo remunerado para las mujeres rurales. Los hatillanos reciben pagos mensuales de compensación de las compañías multinacionales. Cada hogar, independientemente de su tamaño, recibe 300.000 pesos, lo que equivale a unos 80 euros. Además, las empresas les ofrecen un "mercado", es decir, una cierta cantidad de productos alimenticios básicos. Estos consisten principalmente en productos secos, como latas de arroz, pasta o atún (Holguín, 2011). Comparado con los alimentos que consumían antes - sobre todo vegetales, frutas o carne fresca-, esta alimentación es mucho menos saludable y lleva poco valor nutritivo.

En el momento en que se realizó la entrevista con la Mujer 1, estaban algunos de sus 8 niños presentes, jugando afuera de la casa con latas de atún. A la pregunta por quéjuegan con ellas, la madre respondió que a ellos no les gusta el atún, sino el pescado fresco. Se considera esta afirmación como un ejemplo que demuestra que la ayuda que le llega a la Mujer 1 no está ajustada suficientemente a las necesidades específicas de alimentación de ella y sus niños. Se recomienda que los programas de ayuda por parte del Gobierno y las ONG ofrezcan una alimentación más saludable que sea ajustada a las costumbres alimentarias de la comunidad. Adicionalmente, 
la Mujer 4 explica que la ayuda económica que las personas de El Hatillo reciben no es suficiente: "Porque ya lo que dan a uno no alcanza [...], tiene que buscar de otra manera". Entonces muchas mujeres rurales buscan un trabajo que les genere un ingreso financiero.

Según Bermúdez Rico et al. (2014), muchas mujeres se ven obligadas a buscar trabajos mal pagos bajo condiciones precarias. Algunas mujeres de El Hatillo trabajan hoy en concesionarios en las aldeas cercanas. Muchas trabajan con "plata de interés", dinero prestado. Sin embargo, el beneficio que las mujeres obtienen de estas actividades es pequeño. Muy pocas mujeres en zonas rurales tienen la oportunidad de estudiar por falta de dinero, porque deben cuidar a sus familias y porque las universidades están lejos de la casa. La única mujer entrevistada y cuya situación económica es buena, es la lideresa (Mujer 4), que ha estudiado.

Un reto grave con el cual se ven enfrentadas especialmente las mujeres solteras o aquellas cuyas parejas trabajan es que no pueden ejercer una labor fuera de la comunidad por tener que cuidar a los niños. Por ejemplo, el marido de la Mujer 1 trabaja 12 horas al día fuera de la comunidad. Aunque su situación económica implica que ella tendría que trabajar también, prefiere quedarse con sus hijos para no dejarlos solos: "Para mí yo digo que dejar a mis hijos solos todo un día por lo menos solamente que sea una urgencia [...], pero no me parece correcta una cosa así" (3 de mayo de 2017). Por esto, un trabajo en la minería no le llama la atención. La Mujer 3 añade que las mujeres que viven solamente del sueldo de su marido pasan necesidad: "Por allí [con] lo poquito que el marido trabaja [...] con esto mantienen la familia. Y por eso le digo: hambre" ( $4 \mathrm{de}$ mayo de 2017).

Entonces, las mujeres rurales enfrentan una situación muy complicada: no solo no pueden ejercer los trabajos que hacían antes de la llegada de la minería, sino que las posibilidades de encontrar un trabajo remunerado son muy limitadas. Es difícil para ellas cumplir la responsabilidad como cuidadoras, al mismo tiempo que trabajan. Por este motivo, es necesario construir nuevos proyectos de productividad dentro de la misma comunidad.

\section{Pocas posibilidades de trabajo}

Solo unas mujeres encuentran empleo en la minería de carbón. En 2006, el porcentaje de mujeres empleadas en el sector del carbón en Colombia era solo del 19,8 \% (Bermúdez Rico et al., 2011). En La Guajira, únicamente el 2,9 \% de todos los empleados trabajan en la industria minera. Especialmente las mujeres suelen estar desempleadas. En los siete municipios que Romero Epiayú y Barón Romero (2013) estudiaron como parte de su investigación, solo dos mujeres trabajaban en la industria minera. La Mujer 3 de Hatillo explica que el trabajo en las minas es muy exigente: "Es que las mujeres ahora aquí han tenido que trabajar muy duro, las que han podido entrar a las minas. Esto ha sido un impacto duro, porque las mujeres estaban acostumbradas a estar en su casa" (4 de mayo de 2017).

Los requisitos de ingreso al sector son altos. Incluso para tareas menos exigentes, las empresas esperan una carrera profesional. Sin embargo, la mayoría de las mujeres rurales no puede cumplir con este requisito como ya mencionamos. Según una mujer entrevistada, solo un miembro de la familia puede trabajar en la industria minera. La lideresa de El Hatillo afirma que los hatillanos han protestado para ser escuchados por las compañías, principalmente a través de un bloqueo de las vías.

Luego crearon un diálogo organizado con mesas de trabajo. Estas mesas han tenido un efecto positivo, cuenta la lideresa. Dado que las compañías empezaron a capacitar a la población para que tenga el perfil necesario y pueda trabajar en las minas. Sin embargo, hay muy poca gente que consigue un puesto de trabajo, aunque sea capacitada. La Mujer 4 cuenta: "Capacitamos 20 más o menos para trabajar en el área administrativo y a ninguno de ellos les empleaban” (3 de mayo de 2017). La minería puede llegar a tener un efecto negativo para la conformación del tejido social, porque beneficia a unos y a otros no: "El marido de mi vecina tiene un buen trabajo y cambian las relaciones, porque ya el marido trabaja en la minería y el mío 
no" (3 de mayo de 2017). A pesar de las conversaciones, el número de empleadas sigue siendo bajo. En mayo de 2017, solo ocho mujeres de El Hatillo trabajaban en la minería del carbón, según las mujeres entrevistadas.

\section{Desigualdad de género en el sector}

La cuarta hipótesis de este estudio es que existe desigualdad de género dentro del sector minero. En la región de La Guajira, Romero Epiayú y Barón Romero (2013) han podido identificar que no es suficiente aumentar el número de mujeres rurales que trabajan en la minería para mejorar su situación económica. A las mujeres se les paga menos que a los hombres, trabajan en ámbitos que requieren menos formación y sus oportunidades de carrera son limitadas y los contratos de menor duración (Romero Epiayú y Barón Romero, 2013). Bermúdez Rico et al. (2011) muestran en sus estudios que las actividades de extracción de carbón están claramente separadas por género. Mientras que los hombres normalmente realizan trabajos físicos pesados, las mujeres generalmente son contratadas para limpiar o cocinar. Estas suposiciones pueden ser verificadas por los resultados que se han obtenido por las entrevistas cualitativas. Cuando se realizaron las entrevistas en El Hatillo, solo dos mujeres trabajaban como "camioneras" en la industria minera, el resto de ellas se dedicaba a lavar ropa o a la limpieza. Las mujeres entrevistadas cuentan que aquellas que estaban contratadas como limpiadoras recibían alrededor de 860.000 pesos al mes, un poco menos que 300 euros.

Según Barón (2013), la minería del carbón impulsa la "feminización de la pobreza", debido a los aspectos anteriores y Tobón Olarte (2015) cree que refuerza los estereotipos de género prevalecientes en la sociedad.

\section{Discusión}

En este trabajo, se demostró que la minería de carbón a gran escala en Colombia conlleva múltiples efectos negativos para las comunidades, especialmente para las mujeres rurales. Los esfuerzos que las empresas carboníferas han mostrado hasta ahora para ayudar a las mujeres no son suficientes. Por ejemplo, las capacitaciones se presentan como una ayuda a corto plazo. Existe un marco legislativo que debe proteger y asegurar los derechos de las comunidades, pero las leyes y artículos no se cumplen.

Este trabajo muestra que es necesario investigar, analizar y visibilizar más los efectos que la minería genera en la situación económica de las mujeres, y pensar cómo puede mejorarse a largo plazo. Las cuatro hipótesis que se han presentado como resultado de este trabajo muestran tendencias sobre la realidad a la que las mujeres rurales de Colombia se ven confrontadas.

En muchos otros países, los autores y las ONG han observado efectos similares a los que se han presentado en este trabajo. La ONU Mujeres investiga sobre desigualdades de género en el sector extractivo de África. En su informe Gender Equality in the Extractive Industries in Africa (2009) surgen muchos aspectos que se han mencionado en este trabajo.

Un aspecto que aún no se ha mencionado en el contexto colombiano es un posible vínculo entre la expansión de la actividad extractiva y el aumento del trabajo sexual en las áreas respectivas (ONU Mujeres, 2019). Kathy Jenkins (2014) analiza la situación de las mujeres en diferentes partes del Sur global que viven cerca de proyectos extractivos. Ella apoya la tesis de ONU Mujeres:

[...] it is evident that mining camps and compounds, and communities near mining projects, provide an opportunity for women to sell sexual services, as well as attracting women who migrate from other areas in order to find work as sex workers. ${ }^{[7]}$ (p. 335)

Estos estudios dan una razón para examinar este tema en futuras investigaciones sobre Colombia y otros países, con el objetivo de evitar dichas circunstancias. 
En muchas partes del Sur global se puede observar que las mujeres están en desventaja en términos de empleo en la industria minera: "Worldwide it is extremely rare to find any companies with higher than $10 \%$ female employment, with many being less than 5\%" ${ }^{[8]}$ (Eftimie et al., 2009, p. 9). Según Eftimie et al. y ONU Mujeres, no solo son los estereotipos prevalentes específicos de género, sino también el aspecto de que en muchos países se considera desafortunado cuando una mujer ingresa en la industria minera. Las organizaciones están comprometidas a hacer más investigaciones sobre las desigualdades de género en la minería, porque creen que la situación económica de las mujeres puede fortalecerse de esta manera (Eftimie et al., 2009; ONU mujeres, 2019).

Comparar los efectos que la minería tiene en diferentes países y crear métodos de confrontarlos ayudaría no solamente a mejorar la situación de las mujeres rurales en muchos lugares, sino también la de los hombres. Si la mujer no tiene suficientes posibilidades de conseguir un trabajo remunerado para sostener la familia, también tendrá un efecto negativo para los hombres.

Se recomienda diversificar la economía y hacer uso del conocimiento de las mujeres con respecto a la agricultura y a su función de cuidadoras. Es necesario que los gobiernos intensifiquen sus esfuerzos para el desarrollo productivo de las mujeres y los hombres que viven alrededor de las minas.

También es necesario valorar más el trabajo de las mujeres como cuidadoras. Como se ha mostrado en este trabajo, el sistema capitalista beneficia solamente a cierta parte de la población y valora sobre todo trabajos remunerados. El hecho de que la minería destruye la agricultura y que al mismo tiempo da pocas posibilidades de trabajo para la gente local, genera las siguientes preguntas: ¿qué esfuerzos y herramientas son necesarias para que las comunidades puedan aprovechar del desarrollo económico que la minería conlleva para los países? ¿Cómo se puede redistribuir la riqueza y qué formas alternativas de ingreso y productividad existen? Para responder estas y otras preguntas, se considera que el trabajo con las comunidades es fundamental. Conocer las percepciones de las comunidades, en este caso de las mujeres, sus hábitos, sus sueños y su situación familiar, son aspectos que deberían ser considerados en las políticas y los procesos de toma de decisión a nivel nacional y regional.

Las decisiones en esta temática basadas solo en las ventajas económicas nacionales no deben ser el único referente y considerar solo este punto de vista genera el tipo de problemáticas que han sido identificadas en esta investigación. Los gobiernos deben acercarse más a las problemáticas locales y generar estrategias diferenciadas para la solución de estas. El Estado colombiano parece mantenerse alejado de los asuntos, dejando la responsabilidad principal de la minería del carbón a las empresas. Es importante mejorar el diálogo y la cooperación entre los diferentes actores y niveles de gobierno (local, regional y nacional) para atender estas preocupaciones.

\section{Conclusiones}

El objetivo de este trabajo ha sido dar una respuesta a la pregunta: ¿de qué manera la minería legal de carbón a gran escala cambia la situación económica de las mujeres rurales en Colombia? Para esto, se han analizado estudios anteriores y se han realizado entrevistas cualitativas con cinco mujeres de la comunidad El Hatillo en Cesar.

Primero, se demostró que predomina una estrategia de desarrollo en Colombia basada en productos primarios. El sector extractivo ha crecido significativamente en los últimos treinta años. Esto se demuestra en los indicadores macroeconómicos, como el aumento de la producción y las cifras de exportación. El papel del Estado como promotor activo de la producción de carbón está consagrado en la ley. Mientras tanto, las compañías internacionales de carbón están destruyendo importantes fuentes de alimentos de las comunidades rurales. Este es un desafío particular para las mujeres, ya que su trabajo es garantizar la seguridad alimentaria 
de sus familias. En general, es evidente que las mujeres rurales de hoy tienen una mayor responsabilidad de cuidar de su familia, que antes de la llegada de la minería del carbón.

Se pudo demostrar que la economía a gran escala cambia la situación económica de las mujeres rurales en Colombia y esto se ve reflejado en: (i) la economía de subsistencia; (ii) las relaciones de trueque entre vecinos; (iii) la aparición de diferentes estatus sociales por trabajar en el sector minero; (iv) la necesidad de tener varios trabajos y descuidar las labores familiares; (v) la desvalorización del trabajo como cuidadoras; (vi) la disminución de los trabajos tradicionales, debido a la disminución de la fertilidad del suelo, la contaminación del agua, del aire y la desviación de ríos.

La investigación sobre esta temática se encuentra en sus primeras etapas. Debido a la limitada base empírica, se han incluido estudios que las ONG han publicado. Las entrevistas cualitativas intentaron crear una imagen lo más objetiva posible de la realidad social de las mujeres rurales. Sin embargo, la mayoría de las mujeres mostraron una actitud crítica hacia la minería del carbón, por lo que el ambiente era relativamente unilateral. Solo una mujer de El Hatillo dijo que le gustaría trabajar en la minería del carbón.

Por razones de seguridad, tampoco fue posible hablar con los empleados de la minería del carbón en Colombia. Tampoco se ha incluido la perspectiva de los hombres en la investigación. En otro proceso de investigación, sería interesante comparar el impacto económico de la minería del carbón en hombres y mujeres campesinos. El presente trabajo debe entenderse como un primer acercamiento a la opinión general.

Los resultados de este trabajo muestran que la minería del carbón tiene una fuerte influencia en la situación económica de las mujeres rurales en Colombia. Como conclusión, hay un motivo para ubicar los efectos específicos de las actividades extractivas en las mujeres rurales, más en el foco de los debates teóricos y de desarrollo en el siglo XXI.

\section{Referencias}

Acosta, A. (2016). Post-extractivismo: entre el discurso y la praxis. Algunas reflexiones gruesas para la acción. Ciencia Politica, 11(21), 287-332. https://doi.org/10.15446/cp.v11n21.60297.

Arbeitsgruppe Schweiz Kolumbien. (2011). Profite für die Unternehmen, Verarmung für die lokalen Dorfgemeinschaften. Recuperado de http://www.askonline.ch/fileadmin/user_upload/documents/Thema_Wi rtschaft_und_Menschenrechte/Bergbau_Rohstoff/Glencore_Kolumbien/Factsheet_El_Hatillo_-_Glencore. $\mathrm{pdf}$

Barón, D. (Octubre de 2013). Impactos de la minería en los derechos de las mujeres rurales en Colombia. Ponencia presentada en la reunión sobre Mujeres Rurales del Comité Cedaw. Bogotá: Cinep.

Bautista-Revelo, A. J., y Plazas-Mendieta, L. (2018). Tensiones entre la politica extractivista y la restitución de tierras y los derechos territoriales. Colombia: Fundación Comité de Solidaridad con los Presos Políticos.

Bebbington, A. (2015). Political ecologies of resource extraction: Agendas pendientes. European Review of Latin American and Caribbean Studies, 100(100), 85-98. https://doi.org/10.18352/erlacs.10121

Bermúdez Rico, R. E., Rodríguez Maldonado, T., y Roa Avendaño, L. T. (2011, octubre). Mujer y Minería. Ámbitos de análisis e impactos de la minería en la vida de las mujeres: Enfoque de derechos y perspectiva de género. En el Encuentro Latinoamericano Mujer y Mineria. Recuperado de http://www.indepaz.org.co/wp-content/uploa ds/2013/04/Mujer_y_Mineria.pdf

Bermúdez Rico, R. E., Zambrano, K. I., y Roa Avendaño, L. T. (2014). Los territorios, la minería y nosotras: las mujeres nos preguntamos. Guia de trabajo. Colombia: Censat Agua Viva. https://doi.org/10.13140/2.1.2118.7206

Blatter, J. K., Janning, F., y Wagemann, C. (2009). Qualitative Politikanalyse. Eine Einführung in Forschungsansätze und Methoden. Politische Vierteljahresschrift, 50(3), 687-689. Recuperado de http://www.jstor.org/stable/242 01715 . 
Bohórquez Rozo, I. A. (2014). Normatividad para empresas de explotación de carbón en Colombia, sus efectos sociales y ambientales. Bogotá: Universidad Militar Nueva Granada. Recuperado de https://repository.unimilitar.edu.co /handle/10654/12015.

Brand, U., y Dietz, K. (2014). (Neo-)extractivism as development option? Towards current dynamics and contradictions of resource based development in Latin America. Politische Vierteljahresschrift, 298418384(48), 128.

Burchardt, H.-J., y Dietz, K. (2012). Extraktivismus in Lateinamerika - der Versuch einer Fundierung. En H. J. Burchardt, K. Dietz, y R. Öhlschläger (eds.), Umwelt und Entwicklung im 21. Jahrhundert: Impulse und Analysen aus Lateinamerika (1.. ed., pp. 181-200). Baden-Baden: Nomos Verlagsgesellschaft mbH y Co. KG. https://do i.org/10.5771/9783845242880-181

Cedaw. (2019). Primer Informe Sombra especi\#fico de Mujeres Rurales y Campesinas en Colombia. Presentado en la 720 sesio\#n del Comite\# de la Convencio\#n para la eliminacio\#n de todas las formas de discriminacio\#n contra la mujer. Recuperado de http://www.semillas.org.co/es/publicaciones/primer-informe-sombra-especi-769-fico-d e-mujeres-rurales-y-campesinas-en-colombia-presentado-a-la-72o-sesio-769-n-del

Centro de Investigación y Educación Popular (Cinep).(2012). Informe Especial. Minería, Conflictos Sociales y Violación de Derechos Humanos en Colombia. Bogotá. Recuperado de https://www.alainet.org/images/IE_CINEP_octu bre_2012.pdf

Centro de Investigación y Educación Popular (Cinep). (2014) Transformaciones territoriales en la comunidad de El Hatillo. Un recorrido por los impactos de la minería de carbón. Recuperado de https://issuu.com/cinepppp/doc s/cartilla_el_hatillo_web

Centro de Investigación y Educación Popular (Cinep). (2019). Colombia presenta el Primer informe sombra de mujeres rurales y campesinas ante la Cedaw. Recuperado de https://www.cinep.org.co/Home2/component/k2/664-pri mer-informe-sombra-de-mujeres-rurales-y-campesinas-en-colombia.html

Cepalstat. (2017). Colombia: NATIONAL NATIONAL ECONOMIC PROFILE. Recuperado de https://estadistic as.cepal.org/cepalstat/Perfil_Nacional_Economico.html?pais=COL\&i

Comisión Económica para América Latina y el Caribe (Cepal) y Naciones Unidas. (2016). La Inversión Extranjera Directa en América Latina y el Caribe 2016. Cepal. Recuperado de https://repositorio.cepal.org/bitstream/han dle/11362/40213/7/S1600664_es.pdf.

Departamento Nacional de Planeación (DNP). (2011). Plan Nacional de Desarrollo de Colombia 2010-2014. Recuperado de http://www.alcaldiabogota.gov.co/sisjur/normas/Norma1.jsp?i=43101.

Departamento Nacional de Planeación (DNP). (2018). Plan Nacional de Desarrollo 2018-2022. Pacto por Colombia. Pacto por la Equidad.

Drummond. (2019). Procesos de Reasentamiento de Plan Bonito, El Hatillo y Boquerón. Recuperado de http://www.drummondltd.com/responsabilidad-social/proceso-de-reasentamiento/procesos-de-reasentam iento-de-plan-bonito-el-hatillo-y-boqueron/\#estatus-proceso

Eftimie, A., Heller, K., y Strongman, J. (2009). Gender Dimension of the Extractive Industries. Washington, D. C.: The World Bank. Recuperado de http://siteresources.worldbank.org/EXTOGMC/Resources/eifd8_gender_equit y.pdf.

Farah, M. A., y Pérez, E. (2004). Mujeres rurales y nueva ruralidad. Cuadernos de Desarrollo Rural, (51), 137-160. ht tps://doi.org/10.23873/2074-0506-2017-9-3-192-210

Fuentes, G. (2014). El Hatillo, Cesar, Colombia. Recuperado de https://www.flickr.com/photos/103682584@N05 $/ 13946050102$

García, E. (2012). Ecofeminismos Rurales: Mujeres por la Soberanía Alimentaria. Soberanía Alimentaria Biodiversidad y Culturas, 1-44. Recuperado de https://www.soberaniaalimentaria.info/images/estudios/ecofeminismos.pdf.

González Serrano, M.X. (2013). Las transformaciones culturales y territoriales ocasionadas por la minería a gran escala el caso de la comunidad de Boquerón (Cesar-Colombia) (Tesis de maestría). Pontificia Universidad Javeriana, Bogotá. Recuperado de http://hdl.handle.net/10554/15290

Google Earth. (2019). [Mapa de la comunidad El Hatillo]. 
Gudynas, E. (2013). Extracciones, Extractivismo y Extrahecciones. Un marco conceptual sobre la apropiacion de recursos naturales. Observatorio del desarrollo, (18), 1-18. Recuperado de https://www.researchgate.net/public ation/281748932\%0AExtracciones

Hawkins, D. (2014). El carbón y el trabajo en Colombia: a la sombra de la locomotora minera. En La minería de carbón a gran escala en Colombia: impactos económicos, sociales, laborales, ambientales y territoriales (pp. 23-44). Revista Análisis, 68. Recuperado de http://library.fes.de/pdf-files/bueros/kolumbien/11067.pdf

Helfferich, C. (2011). Die Qualität qualitativer Daten. Manual für die Durchführung qualitativer Interviews. Verlag für Sozialwissenschaften.

Holguín, G. (2011). Caracterización del caso El Hatillo. Actores, dinámicas y conflictos. Pensamiento y Acción Social. Recuperado de https://studylib.es/doc/6458110/caracterizaci\%C3\%B3n-del-caso-el-hatillo

Jenkins, K. (2014). Women, mining and development: An emerging research agenda. The Extractive Industries and Society, 1(2), 329-339. https://doi.org/https://doi.org/10.1016/j.exis.2014.08.004:

Kruse, J. (2015). Qualitative Interviewforschung: ein integrativer Ansatz. Weinheim: Beltz Juventa.

Ministerio de Minas y Energía (Minenergía). (2019). PINE - Cesar. Recuperado de https://www.minenergia.gov.co /pine-cesar

Minminas, y Agencia Nacional de Minería (ANM). (2017, 16 de mayo). Producción de carbón en ascenso. Recuperado de https://www.anm.gov.co/?q=produccion_de_carbon_en_ascenso_principal

Observatorio de Conflictos Ambientales (OCA). (2019). Conflicto: Minería de Carbón - Drummond - PRODECO Murray Energy - Cesar. Recuperado de https://conflictos-ambientales.net/oca_bd/env_problems/view/4.

Olivares Rivera, J. L., y Manrique Galvis, J. J. (2018). Producción y exportaciones de carbón en Colombia. Minminas. Recuperado de https://www.minenergia.gov.co/documents/10192/23966843/301118_prod_expo_carbon_ii i_trim_2018.pdf/630e9cb8-32cf-41e3-b6ea-18564d35320d.

ONU Mujeres. (2019). Gender Equality in the Extractive Industries in Africa. Recuperado de https://www.goo.gl/JB vMcYcontent_copy.

Osorio, C. (2011, 11 de septiembre). Hay locomotora minera, pero no locomotora para la reubicación de los vecinos. La Silla Vacia. Recuperado de https://lasillavacia.com/historia/hay-locomotora-minera-pero-no-locomotora-p ara-la-reubicacion-de-los-vecinos-27427

Prodeco (2019). Reasentamiento. Recuperado de http://www.grupoprodeco.com.co/es/sostenibilidad/reasentamien to

Romero Epiayú, J., y Barón Romero, D. (2013). Impacto de la explotación minera en las mujeres rurales: afectaciones al derecho a la tierra y el territorio en el sur de La Guajira, Colombia. Cinep. Recuperado de http://www.oidhaco. org/uploaded/content/article/2091974838.pdf

Sociedad Geográfica de Colombia [Sogeocol]. (2017). Geografia de Colombia, Departamentos de Colombia, Cesar. Recuperado de https://www.sogeocol.edu.co/cesar.htm.

Suhner, S. (19 de noviembre de 2019). Die Umsiedlung von El Hatillo gerät erneut in Probleme. Recuperado de https: //www.askonline.ch/themen/die-umsiedlung-von-el-hatillo-geraet-erneut-in-probleme.

Tobón Olarte, G. (2015). Mujeres, conflictos socioambientes y resolución 1325 de las Naciones Unidas. Bogotá: Red Nacional de Mujeres.

Unidad de Planeación Minero Energética (UPME). (2006). Colombia País Minero - Plan Nacional para el Desarrollo Minero visión al año 2019. Recuperado de http://bdigital.upme.gov.co/handle/001/906.

Unidad de Planeación Minero Energética (UPME). (2018). Producción, regalias y comercio exterior. Recuperado de ht tp://www1.upme.gov.co/simco/Cifras-Sectoriales/Paginas/Informacion-estadistica-minera.aspx

Urbina Sánchez, E., Cita Triana, R. A., y Campo Robledo, J. A. (2016). Explotacion de carbono y desplazamiento tensiones entre derechos y procesos economicos. Bogotá: Universidad Católica de Colombia. https://doi.org/10.1 017/CBO9781107415324.004

YoSoyMujerRural. (2019). YoSoyMujerRural. Recuperado de http://www.yosoymujerrural.com/ 


\section{Notas}

* Artículo de investigación.

Este artículo es resultado del trabajo titulado "Las consecuencias económicas de la extracción de carbón legal en Colombia para mujeres rurales. Estudio de caso sobre la situación en El Hatillo, departamento El Cesar". Fue realizado por la Universidad Heinrich-Heine de Düsseldorf y la Universidad Católica de Colombia, en el periodo de febrero a junio de 2017.

[1] En alemán: Rohstoffboom.

[2] El término 'extractivismo' (clásico) se refiere a países con gobiernos neoliberales autoritarios como Colombia. Ellos impulsan la extracción de materias primas para así fortalecer la economía de sus países. Para lograr este objetivo, promulgan nuevas leyes o modifican las que ya existen. En el neo-extractivismo, el Estado juega un papel más activo e invierte una parte de los ingresos adicionales en programas sociales (Gudynas, 2013). Unos autores también hablan del post-extractivismo (Acosta, 2016).

[3] Se puede diferenciar entre mujeres rurales campesinas, indígenas y afrodescendientes. En este trabajo nos enfocamos en las mujeres rurales campesinas. Entendemos que ellas viven en regiones rurales y que su situación económica está basada en la agricultura en su mayor parte.

[4] Ver en Anexos del Informe Sombra crónica: Mujeres rurales del Guaviare en relación con la implementación del Acuerdo de Paz (Cedaw, 2019).

[5] Traducción del término al español: principio de nieve. Este funciona de tal manera de que la persona investigadora construye una red de contactos a través de una base de contactos que se va ampliando poco a poco durante la investigación.

[6] Véase https://www.facebook.com/COMUNDO-Colombia-916915715037935/

[7] Traducción al español: "Es evidente que los campamentos y los complejos mineros, y las comunidades cercanas a los proyectos mineros, brindan una oportunidad para que las mujeres vendan servicios sexuales, así como para atraer a mujeres que emigran de otras áreas para encontrar trabajo como trabajadoras sexuales".

[8] Traducción al español: "En todo el mundo es extremadamente difícil encontrar empresas con más del 10\% de empleo femenino, y en muchas la participación está en 5\%".

\section{Licencia Creative Commons CC BY 4.0}

Cómo citar este artículo: Gerstenberg, F., y Villegas González, P. A. (2019). La minería de carbón en Colombia y la situación económica de las mujeres rurales: la comunidad El Hatillo (Cesar, Colombia). Ambiente y Desarrollo, 23(45). https://doi.org/10.11144/Javeriana.ayd23-45.mccs 